\title{
Acute Patellar Tendon Rupture after Total Knee Arthroplasty Revision
}

\author{
Seung Joon Rhee, $\mathrm{MD}^{1}$, The Hien Pham, $\mathrm{MD}^{2}$, and Jeung Tak Suh, $\mathrm{MD}^{1}$ \\ ${ }^{1}$ Department of Orthopedic Surgery, Pusan National University Hospital, Busan, Korea; ${ }^{2}$ Department of Orthopedic Surgery, Nguyen Tri Phuong Hospital, Ho Chi Minh, \\ Vietnam
}

Patellar tendon rupture is a catastrophic complication following total knee arthroplasty (TKA). Though revision TKA has been suspected of being a predisposing factor for the occurrence of patellar tendon rupture, there are few reports on patellar tendon rupture after revision TKA. Here, we present a case of acute patellar tendon rupture that occurred after TKA revision. In the patient, the patellar tendon was so thin and could not be repaired, and accordingly was sutured end to end. We used the anterior tibialis tendon allograft to augment the poor quality patellar tendon tissue. Fixation of the allograft was done by using the bone tunnel created through tibial tuberosity and suturing the allograft to the patellar tendon and quadriceps tendon. The patient was instructed to wear a full extension knee splint and was kept non-weight bearing for 6 weeks after operation. Full knee extension could be achieved 6 weeks postoperatively.

Keywords: Patella, Tendon rupture, Revision, Arthroplasty

Extensor mechanism disruption after total knee arthroplasty (TKA) is an infrequent complication with an incidence of $0.17 \%$ $2.5 \%{ }^{1)}$, but inconsistent results associated with various reported operative treatment techniques imply the challenging nature of this complication. There are many surgical treatment options ranging from nonoperative treatment with a brace or cast immobilization to reconstruction or knee arthrodesis ${ }^{2}$. Patellar tendon rupture is a part of extensor mechanism disruption after TKA. The incidence of patellar tendon rupture after primary TKA is reported from $0.17 \%$ to $1.4 \%{ }^{3)}$ whereas the incidence after revision TKA has not been reported but perceived much higher. The goals of repair of the ruptured patellar tendon include restoring the functioning quadriceps mechanism and allowing good range

Received October 30, 2014; Revised January 17, 2015;

Accepted January 29, 2015

Correspondence to: Jeung Tak Suh, MD

Department of Orthopaedic Surgery, Pusan National University

Hospital, 179 Gudeok-ro, Seo-gu, Busan 602-739, Korea

Tel: +82-51-240-7248, Fax: +82-51-247-8395

E-mail: jtsuh@pusan.ac.kr

This is an Open Access article distributed under the terms of the Creative Commons Attribution Non-Commercial License (http://creativecommons.org/licenses/by-nc/4.0/) which permits unrestricted non-commercial use, distribution, and reproduction in any medium, provided the original work is properly cited. of motion (ROM) of the knee, which are best achieved with early mobilization ${ }^{2)}$. The patellar tendon in TKA revision (TKAR) is often of very poor tissue quality, and either requires augmentation even if its direct repair is possible or reconstruction using autograft, synthetic graft or allograft ${ }^{4)}$. Direct repair and long leg immobilization often fail and lead to re-rupture of the patellar tendon, weakness of the quadriceps muscle or a stiff knee. Therefore, determining the exact cause of patellar tendon rupture and planning proper reconstruction operation for individual patient will lead to better results.

\section{Case Report}

An 83-year-old female patient was admitted with left knee pain and no history of disease. She had osteoarthritis in both knees and underwent bilateral TKA. The left knee was replaced 23 years ago and the right was replaced 7 years ago. The radiograph showed severe femorotibial compartment narrowing in the left knee, indicating severe wearing of the polyethylene insert. Radiolucency extended to the tibial shaft and advanced osteolytic lesion was also visible (Fig. 1). The left knee ROM was $90^{\circ}$ flexion with $20^{\circ}$ flexion contracture. A TKAR was indicated. The knee joint was opened though the previous skin incision using a me- 
dial parapatellar retinacular approach. After radical synovectomy of the thick inflamed, hypertrophic synovium, sufficient joint space was secured to perform surgery without using any revision approach. Inside the joint capsule, severe wearing of the polyethylene insert, metal backed patellar component, and tibial tray was observed and the surrounding synovium became black due to global metallosis (Fig. 2). After removing the worn prosthesis, all the bones and soft tissue infiltrated by metallosis were debrided and bone preparation using morcellized bone allograft was done. Subsequently, a constrained prosthesis (SIGMA TC3; DePuy Synthes, West Chester, PA, USA) with a $22.5 \mathrm{~mm}$ thickness polyethylene was inserted. Neutral alignment of the axis and InsallSalvati (IS) ratio of 0.81 (Fig. 3) (normal between 0.8 and 1.2) (5) $^{\text {) }}$ were confirmed on postoperative radiographs.
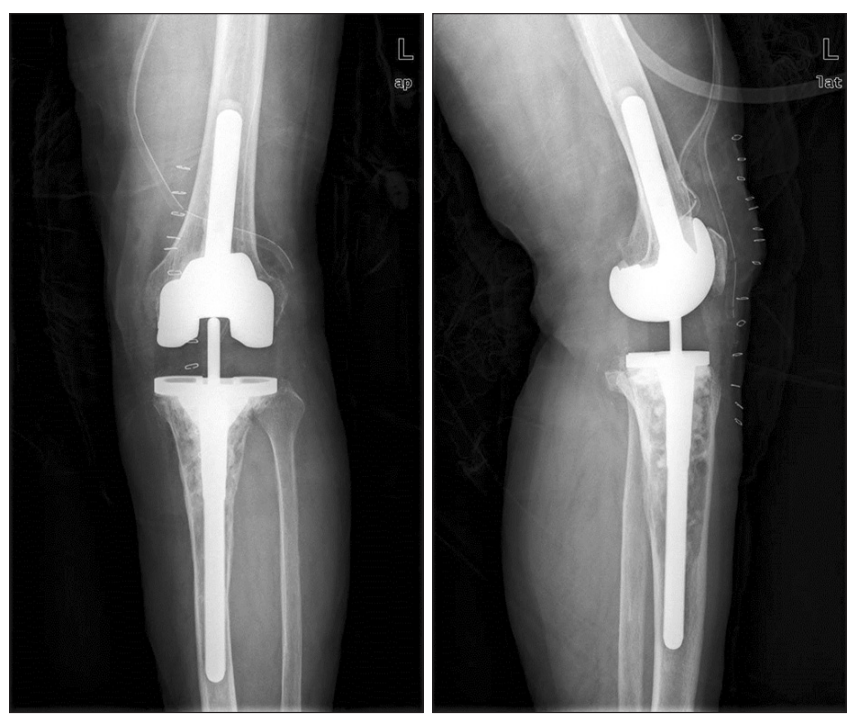

Fig. 3. The Insall-Salvati ratio measured on the radiographs taken after

total knee arthroplasty revision was 0.81 .
Fig. 1. Radiographs obtained prior to total knee arthroplasty revision showed severe femorotibial compartment narrowing.

(n)

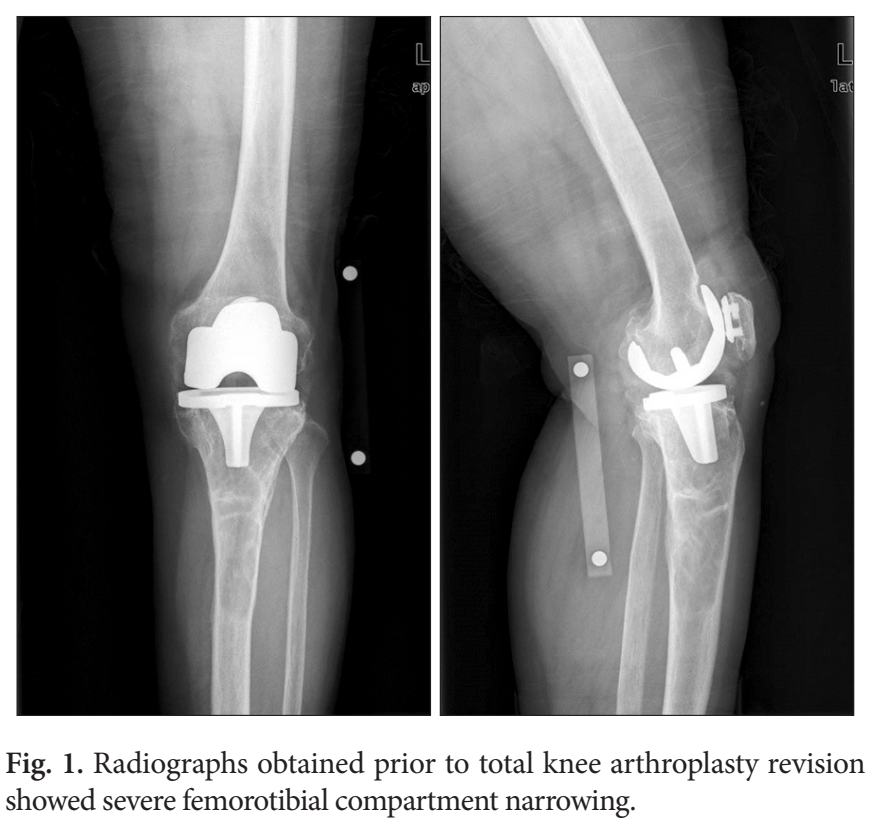

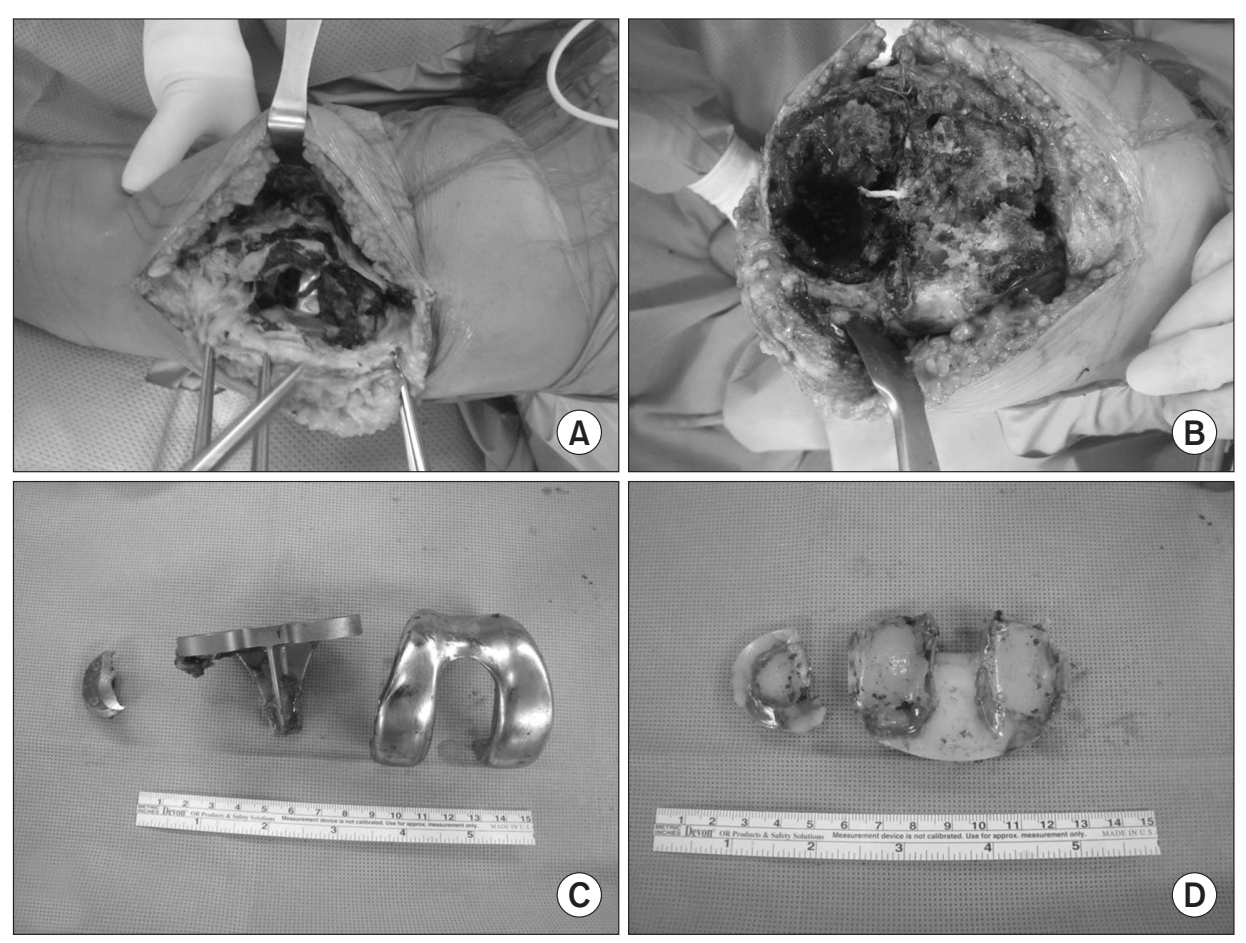

Fig. 2. During total knee arthroplasty revision, severe metallosis was observed in the surrounding synovium (A) and huge bone defect in the proximal tibia caused by osteolysis was observed (B). (C and D) Retrieved implants showed severe wear of the polyethylene insert and metal backed patella and tibia. 
Until 10 days postoperatively, the patient was on her ordinary course of recovery and rehabilitation without any abnormal sign of derangement. Left knee ROM reaching $100^{\circ}$ of flexion starting from full extension was achieved. But, she slipped backward with her left foot still placed on the floor during first ambulation trial, and she could not have her left knee extended afterwards. We checked radiographs of both knees and there was no sign of prosthesis dislocation or fracture, but the IS ratio was 0.51 (Fig. 4$)^{7}$. Ultrasound was performed to assess the patellar tendon, which
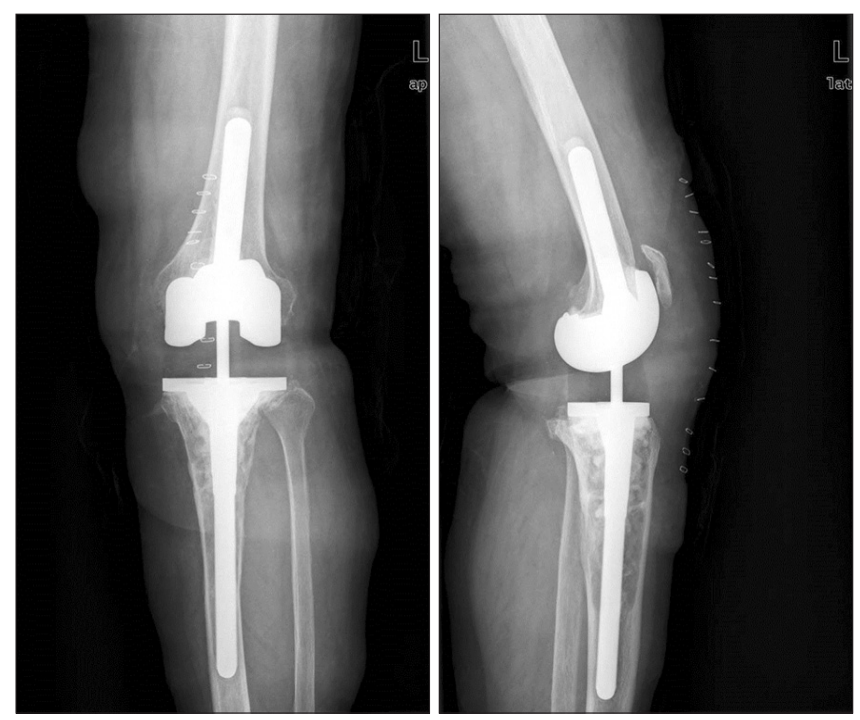

Fig. 4. The Insall-Salvati ratio measured on the radiographs taken after the falling event was 0.51 . showed discontinuity of the patellar tendon at its tibial insertion, implying a patellar tendon rupture (Fig. 5). The patient had another operation for reconstruction of the patellar tendon 2 days after the accident.

The same incision was made along the previous incision scar on the left knee, through which we confirmed the patellar tendon was ruptured at distal $1 / 3$ of its length. After minimal debridement, the free ends of tendon were sutured together by 4 nonabsorbable sutures (Fiberwire; Arthrex Inc., Naples, FL, USA) with the knee in full extension. We used the Krackow whip stitch to prepare the tendon (Fig. 6). Then, we used tibialis anterior allograft tendon of $240 \mathrm{~mm}$ length and $15 \mathrm{~mm}$ width to augment the patellar tendon. An approximately $3 \mathrm{~mm}$ diameter bone tunnel was penetrated at tibial tuberosity in the medial to lateral direction using a $2.8 \mathrm{~mm}$ drill bit. The allograft was taken through this tunnel by pulling with the sutures. Two ends of the allograft tendon were sutured to the patellar tendon and lateral and medial patellar retinacula by nonabsorbable Ethibond 2-0 suture with the knee in full extension. The tension of the allograft was maintained maximum in full knee extension. We used C-arm to prevent patellar baja and fracture. After reconstruction, the tension of the left knee and radiography were examined (Fig. 7). The patient wore a cylindrical splint with the knee in full extension and remained non-weight bearing for 6 weeks. At the last follow-up, 12 months after the patellar tendon reconstruction, the patient could extend the left knee with her own muscle power leaving $10^{\circ}$ of extension lag from $100^{\circ}$ flexion position. But, the IS ratio remained abnormal as 0.53 (Fig. 8).
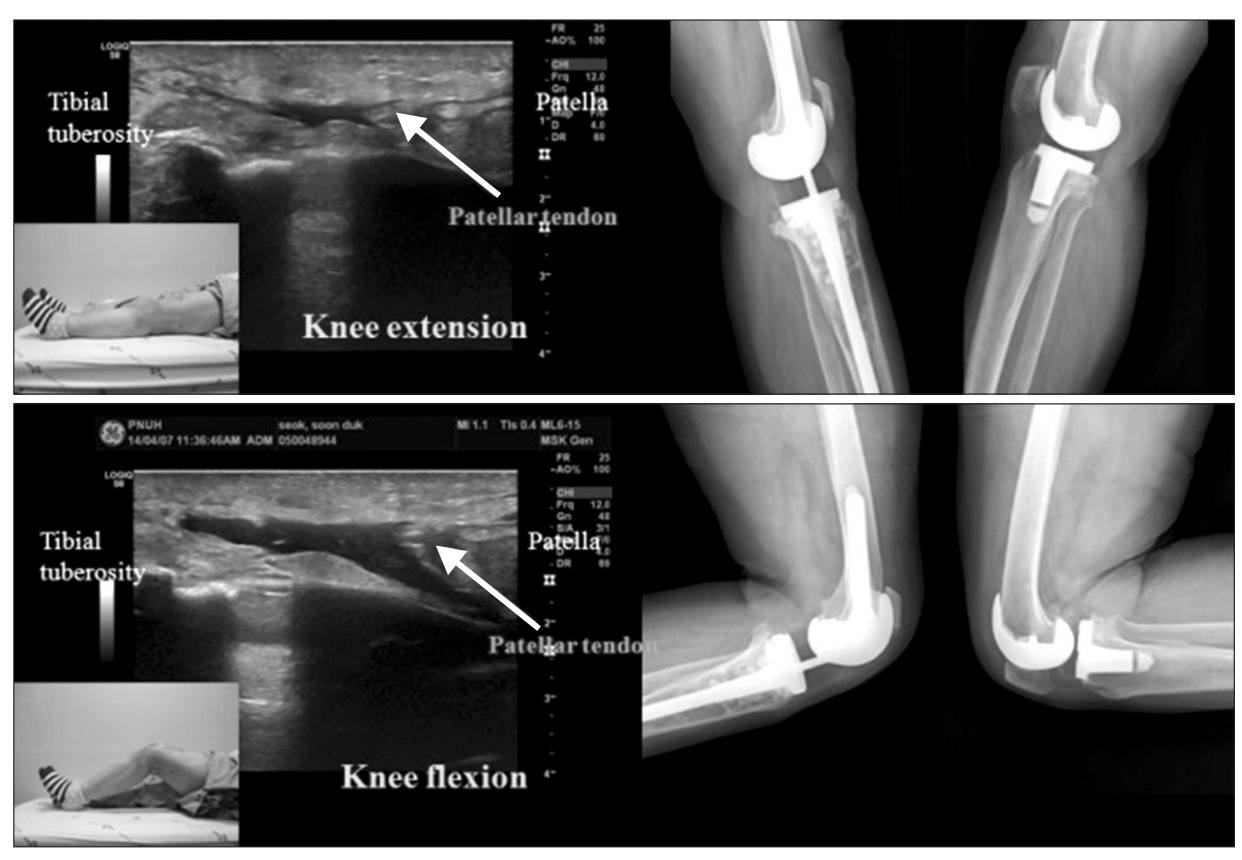

Fig. 5. Ultrasound imaging of the left knee showed defect in the patellar tendon and dynamic radiography of both knee showed prominent upward migration of the patella in the flexion of the knee. 

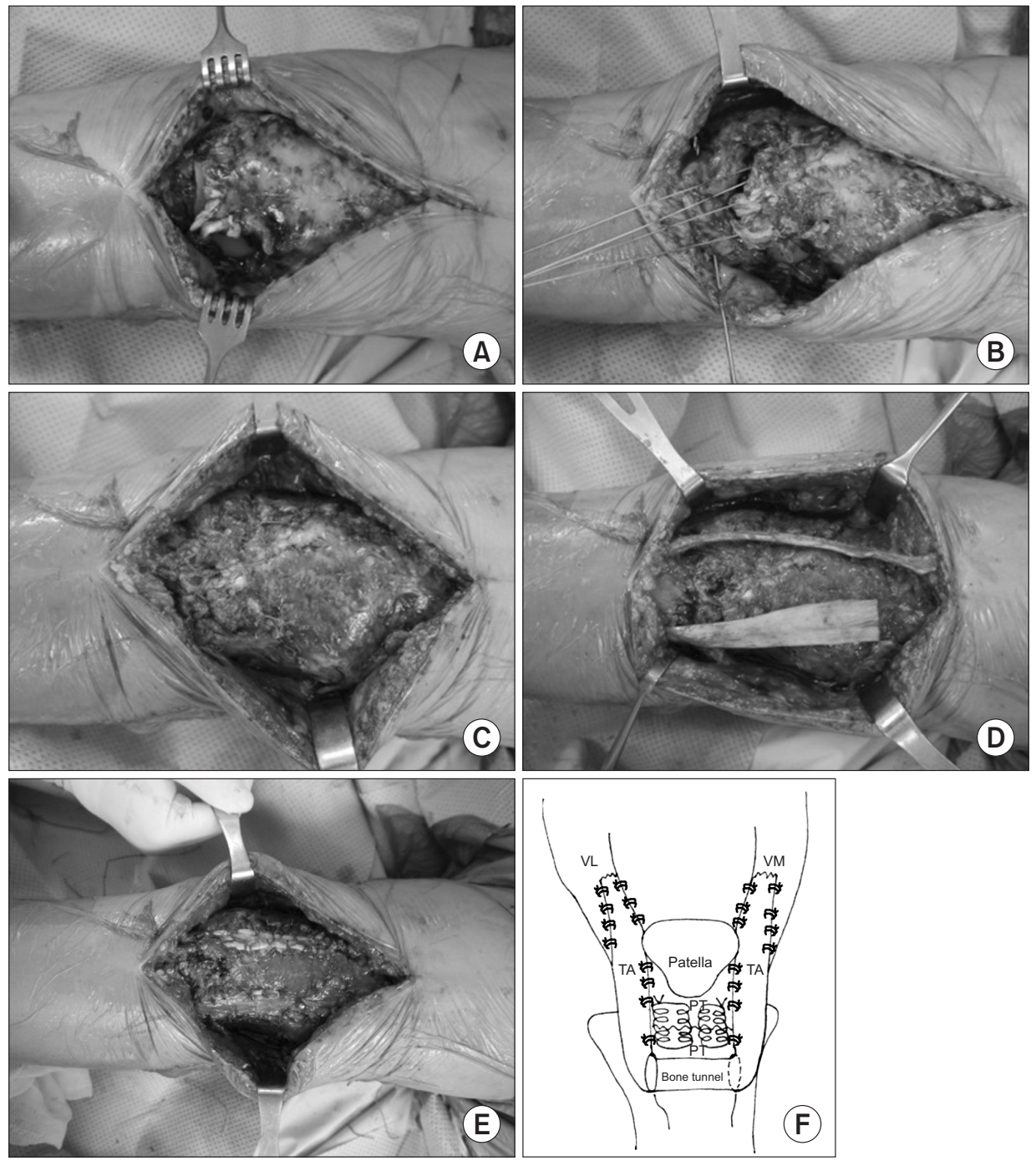

Fig. 6. Intraoperative pictures. (A) A rupture was identified in the distal $1 / 3$ of the patellar tendon. (B) Four-strand Krackow sutures were performed. (C) A tibialis anterior tendon allograft was passed through bone tunnel in the tibial tuberosity. (D) The allograft tendon was sutured in an onlay fashion to the patellar retinaculum and quadriceps tendon. (E) The repair was completed. (F) Schematic drawing of the repair construct. After passing the fourstrand Krackow sutures across the torn ends of the patellar tendon (PT), the tibialis anterior tendon allograft (TA) was passed through the bone tunnel and was sutured side-to-side to the patellar tendon and as an onlay to the patellar retinaculum and quadriceps tendon for augmentation. VL: vastus lateralis, VM: vastus medialis.

\section{Discussion}

In our patient, the initial radiography did not reveal a thin patellar fracture or a patellar avulsion fracture, the mechanical axis was not malaligned, and the implants were in their right position. But, considering her trauma mechanism with sudden hyperflexion injury of the knee and following inability of knee extension, we suspected extensor mechanism disruption. The ultrasound exam showed discontinuity of the patellar tendon and thus an acute patellar tendon rupture was diagnosed. In TKA patients, a patellar tendon rupture can occur intraoperatively, immediately postoperatively, or even thereafter. Excessive tension on the patellar tendon during exposure of a stiff or ankylosed knee can cause avulsion of the tendon from the tibia. The knee prosthesis design and operative technique also play a role in the rupture of the patellar tendon. Some authors reported that malalignment of the component may place excessive stress on the extensor mechanism ${ }^{2}$. In our case, we suspect the poor quality of patellar tendon that was inflamed by the phagocytic activity and osteolysis following metallosis was the main reason of the acute patellar tendon rupture ${ }^{8)}$.

There are many options to reconstruct the ruptured patellar tendon: direct primary repair alone by suturing, stapling, or wiring the tendon to the tibial tuberosity; primary repair combined with biologic or synthetic graft augmentation (e.g., gracillis tendon, free fascia lata graft, plantaris tendon, semitendinosus tendon, and medial gastrocnemius rotational muscle flap); and allograft tissue augmentation with extensor mechanism or Achilles tendon. Patellotibial fusion, knee arthrodesis, and even above-theknee amputation are salvage techniques that may be considered if a surgical repair is not possible or if an extensor mechanism reconstruction fails ${ }^{2}$. Since our patient presented with an acute 

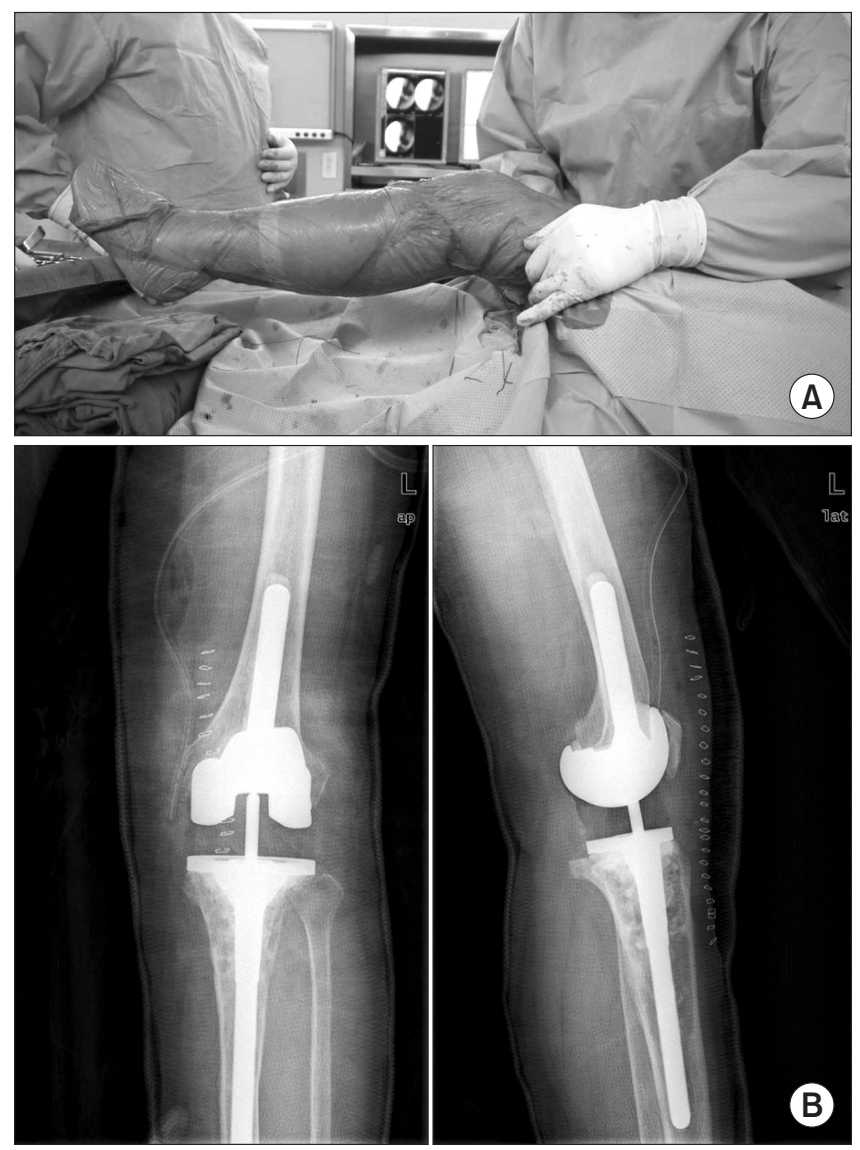

Fig. 7. (A) Pictures taken after surgery showed that the knee could be maintained in extended position. (B) The Insall-Salvati ratio on the postoperative radiographs was measured as 1.19 .

patellar tendon rupture, the first treatment of choice was primary repair by suturing. Primary repair is the best option in acute cases if the tendon tissue is long and durable enough for repair. Rand et al." reported that direct repair of patellar tendon rupture after TKA had variable results in 18 knees: $25 \%$ of the patients had successful outcome and the best result was obtained with staple fixation. However, in our case, the patellar was so thin that we could not perform staple fixation during TKAR to prevent a patellar fracture. We used a tendon graft for augmentation of the repair for better results. The fixation method in the distal place was the tibial tuberosity bone tunnel and the proximal place was suture fixation to the patellar tendon and lateral and medial patellar retinacula.

There are various augmentation materials including autograft, allograft and synthetic graft. Semitendinosus tendon, gracillis tendon, free fascia lata graft, and gastrocnemius rotational flap are commonly used for autograft ${ }^{2}$. In our patient who was 83 years old and had TKA on the right knee and TKAR on the left knee, autograft was not an option considering the risk of donor
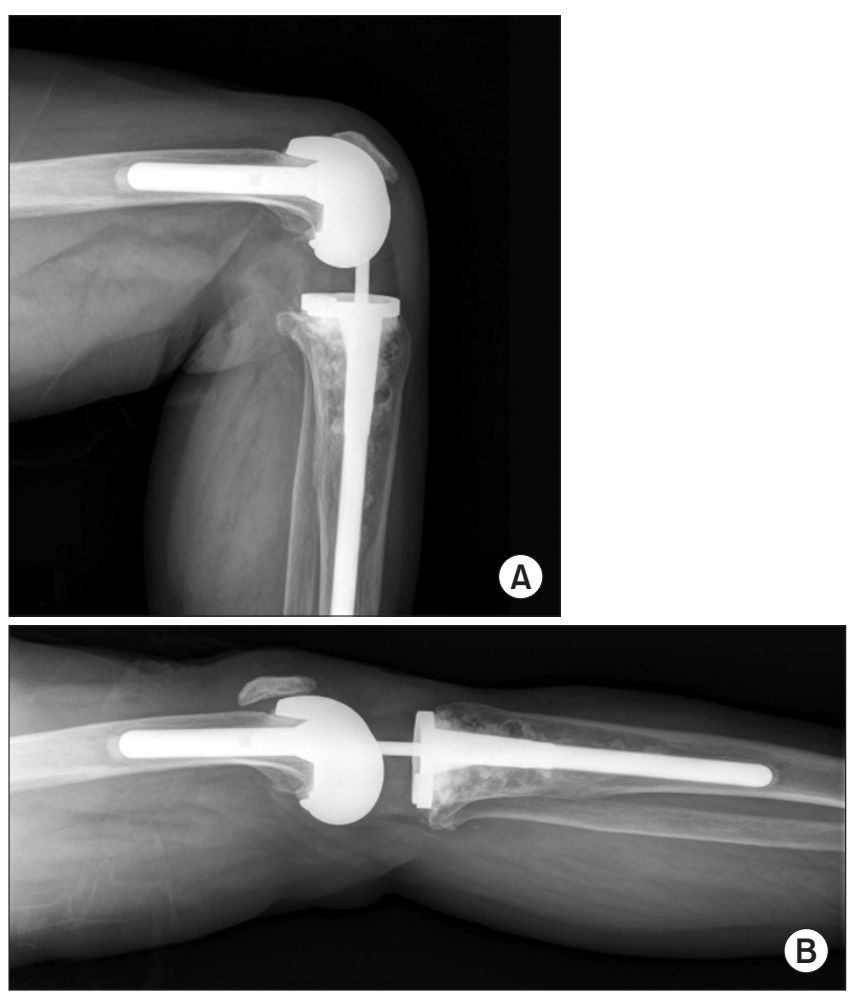

Fig. 8. Twelve-month postoperative radiographs showing $100^{\circ}$ of knee flexion (A) and $10^{\circ}$ of extension lag (B).

site morbidity in elderly TKAR female with poor tissue holding strength. A synthetic graft can be an alternative in such a case because it can be used without the risk of donor site morbidity in TKAR patients with increased risk of infection and poor tissue holding strength. Dacron, Gore-tex polypropylene and other artificial ligaments such as Leeds-Keio ligament can be used as synthetic graft. The loading force after operation can be transferred mainly through the synthetic graft early, and this force is gradually transferred through the biologically adhered graft or healed tendon. In the patient with poor strength of tissue, we could not ascertain the effectiveness of synthetic graft. Allogarft is the best choice for reconstruction in the patient with poor quality host tissue. The advantages of allograft are unlimited supply, various tissue types, absence of donor site morbidity, and superior strength compared to autograft. On the other hand, the disadvantages include immune reaction, disease transmission and poor graft strength $^{2}$. However, disease transmission is rare and can be controlled, and immune reaction can be minimized by using deep fresh frozen allograft ${ }^{2}$.

Regarding the tensioning of the patellar tendon, one should be careful not to apply excessive or insufficient tension to the repaired or reconstructed patellar tendon to avoid patellar baja or alta and a patellar fracture. In 2005, Burnett et al. ${ }^{10)}$ presented 
a study that directly compared the results of reconstruction with extensor mechanism allograft and postoperative rehabilitation using two different techniques. The authors surmised that tensioning of the extensor mechanism allograft in full extension is a critical determinant of success and yields a higher rate of clinical success in terms of restoration of full active extension and better walking status without any adverse impact on flexion. Thus, we fixed the allograft in full extension position under maximum tension and used C-arm to prevent patellar baja.

Patellar tendon rupture after TKA, especially after TKAR, is difficult to be treated with good results and causes confusion in the selection of reconstruction methods. Exact identification of the cause of rupture and appropriate reconstruction planning using proper augmentation methods, fixation techniques and rehabilitation protocols are crucial for overcoming the unexpected complication and obtaining good surgical outcome. In our patient, prior to the occurrence of the rupture, damage to the patellar tendon tissue was suspected due to the inflammatory reaction and osteolysis following metallosis. Therefore, we chose to repair the patellar tendon with an augmentative tibialis anterior tendon allograft that was considered sufficiently strong to hold the whole repair construct. We suggest that the surgeon should pay extraordinary attention to severe wear or osteolysis during TKAR to prevent patellar tendon rupture.

\section{Conflict of Interest}

No potential conflict of interest relevant to this article was reported.

\section{References}

1. Schoderbek RJ Jr, Brown TE, Mulhall KJ, Mounasamy V, Iorio R, Krackow KA, Macaulay W, Saleh KJ. Extensor mechanism disruption after total knee arthroplasty. Clin Orthop Relat Res. 2006;446:176-85.

2. Jarvela T, Halonen P, Jarvela K, Moilanen T. Reconstruction of ruptured patellar tendon after total knee arthroplasty: a case report and a description of an alternative fixation method. Knee. 2005;12:139-43.

3. Fan CH, Lo CK, Yung SH, Chan KM. A rare mode of extensor mechanism failure in total knee arthroplasty. J Arthroplasty. 2011;26:338.

4. Aracil J, Salom M, Aroca JE, Torro V, Lopez-Quiles D. Extensor apparatus reconstruction with Leeds-Keio ligament in total knee arthroplasty. J Arthroplasty. 1999;14:204-8.

5. Blackburne JS, Peel TE. A new method of measuring patellar height. J Bone Joint Surg Br. 1977;59:241-2.

6. Insall J, Salvati E. Patella position in the normal knee joint. Radiology. 1971;101:101-4.

7. Melloni P, Valls R, Veintemillas M. Imaging patellar complications after knee arthroplasty. Eur J Radiol. 2008;65:478-82.

8. Grammatopoulos G, Pandit H, Kamali A, Maggiani F, GlynJones S, Gill HS, Murray DW, Athanasou N. The correlation of wear with histological features after failed hip resurfacing arthroplasty. J Bone Joint Surg Am. 2013;95:e81.

9. Rand JA, Morrey BF, Bryan RS. Patellar tendon rupture after total knee arthroplasty. Clin Orthop Relat Res. 1989;(244):2338.

10. Burnett RS, Berger RA, Della Valle CJ, Sporer SM, Jacobs JJ, Paprosky WG, Rosenberg AG. Extensor mechanism allograft reconstruction after total knee arthroplasty. J Bone Joint Surg Am. 2005;87 Suppl 1:175-94. 